\title{
SAR156597 in idiopathic pulmonary fibrosis: a phase 2 placebo-controlled study (DRI11772)
}

\author{
Ganesh Raghu ${ }^{1}$, Luca Richeldi², Bruno Crestani ${ }^{3,4}$, Peter Wung ${ }^{5}$, \\ Raphael Bejuit ${ }^{6}$, Corinne Esperet ${ }^{6}$, Christian Antoni $^{6}$ and Christina Soubrane ${ }^{6}$
}

Affiliations: ${ }^{1}$ Center for Interstitial Lung Diseases, Division of Pulmonary, Critical Care and Sleep Medicine, University of Washington Medical Center, Seattle, WA, USA. ${ }^{2}$ Fondazione Policlinico Universitario A. Gemelli IRCCS, Università Cattolicà del Sacro Cuore, Rome, Italy. ${ }^{3}$ APHP, Hôpital Bichat, Service de Pneumologie A, Centre de Référence Constitutif des Maladies Pulmonaires Rares, DHU FIRE, Paris, France. ${ }^{4}$ Université Paris Diderot, Sorbonne Paris Cité, INSERM Unité 1152, Paris, France. ${ }^{5}$ Medical Operations, Sanofi R\&D, Bridgewater, NJ, USA. 'Immunology and Inflammation Therapeutic Area, Sanofi R\&D, Paris, France.

Correspondence: Ganesh Raghu, University of Washington Medical Center, 1959 NE Pacific, Campus Box 356175, Seattle, WA 98195-6522, USA. E-mail: graghuduw.edu

@ERSpublications

The phase 2b DRI11772 study failed to demonstrate benefit of anti-IL-4/-13 bispecific antibody SAR156597 in the treatment of idiopathic pulmonary fibrosis http://ow.ly/RAQ130m6ZF6

Cite this article as: Raghu G, Richeldi L, Crestani B, et al. SAR156597 in idiopathic pulmonary fibrosis: a phase 2 placebo-controlled study (DRI11772). Eur Respir J 2018; 52: 1801130 [https://doi.org/10.1183/ 13993003.01130-2018].

ABSTRACT A phase 2b trial (NCT02345070) was conducted to evaluate the efficacy and safety of two dose levels/regimens of SAR156597 (a bispecific IgG4 antibody that binds and neutralises both circulating interleukin-4 and interleukin-13), in comparison with placebo, administered to patients with idiopathic pulmonary fibrosis (IPF) over 52 weeks.

DRI11772 was a multinational randomised double-blind placebo-controlled phase $2 \mathrm{~b}$ trial. Patients aged $>40$ years with a documented diagnosis of IPF received SAR156597 $200 \mathrm{mg}$ once every week (QW), SAR156597 $200 \mathrm{mg}$ once every 2 weeks (Q2W) or placebo, over 52 weeks. The primary efficacy end-point was absolute change from baseline in forced vital capacity (FVC) \% predicted at 52 weeks.

Of 327 randomised patients, 325 received treatment with placebo $(n=109)$, SAR156597 Q2W $(n=108)$ or SAR156597 QW ( $\mathrm{n}=108)$. The mean change from baseline in FVC $\%$ pred at 52 weeks was $-5.8 \%,-5.2 \%$ and $-6.3 \%$ for the placebo, Q2W and QW arms, respectively (Q2W versus placebo, $\mathrm{p}=0.59$; $\mathrm{QW}$ versus placebo, $\mathrm{p}=0.63$ ). The safety profile observed in the three treatment arms was generally similar, although serious adverse events were more common in the QW arm than in the other arms.

The DRI11772 study failed to demonstrate benefit of SAR156597 in the treatment of IPF.

This article has supplementary material available from erj.ersjournals.com

Clinical trials: This study is registered at www.clinicaltrials.gov with identifier NCT02345070. Qualified researchers may request access to patient-level data and related study documents including the clinical study report, study protocol with any amendments, blank case report form, statistical analysis plan and dataset specifications. Patient-level data will be anonymised and study documents will be redacted to protect the privacy of trial participants. Further details on Sanofi's data sharing criteria, eligible studies and process for requesting access can be found at: https://www. clinicalstudydatarequest.com

The results of this study were presented in part at the American Thoracic Society annual meeting 2018. Raghu G, Richeldi L, Crestani B, et al. Safety and efficacy of SAR156597 in idiopathic pulmonary fibrosis (IPF): a phase 2, randomized, double-blind, placebo-controlled study. Am J Respir Crit Care Med 2018; 197: A2441. 


\section{Introduction}

Idiopathic pulmonary fibrosis (IPF) is a progressive, chronic fibrotic disease of unknown cause, characterised by worsening dyspnoea and progressive loss of lung function, with a median survival of 2-3 years from diagnosis $[1,2]$. According to the World Health Organization mortality database, the median mortality rate for IPF in Europe between 2001 and 2013 was 3.75 per 100000 for men and 1.50 per 100000 for women [3]. IPF is characterised by progressive loss of lung function and worsening of symptoms, including dyspnoea and cough; the aims of treatment are to slow disease progression, improve survival and maintain quality of life [2]. Current treatment recommendations are limited to the use of nintedanib (a tyrosine kinase inhibitor), pirfenidone (an anti-fibrotic agent) and antacid medications [4]. While the standard of care has improved over recent years, disease progression continues [2]. Although lung transplantation extends survival, this option is not available for most patients [2]. Therefore, there is a continued unmet medical need for alternative treatment options.

Interleukin (IL)-4 and IL-13 may represent rational therapeutic targets for IPF. In patients with IPF, IL-4 and IL-13 levels in bronchial alveolar lavage fluid are elevated compared with normal controls [5, 6]. IL-4 and IL-13 can exhibit significant pro-fibrotic activity through the recruitment, activation and proliferation of fibroblasts, macrophages and myofibroblasts [7, 8], and by elevating periostin expression [9]. Monoclonal antibodies to IL-13 have been investigated in phase 2 IPF trials (tralokinumab (MedImmune LLC, Gaithersburg, MD, USA), NCT01629667 [10]; lebrikizumab (Hoffmann-La Roche, Basel, Switzerland), NCT01872689; QAX576 (Novartis, Basel, Switzerland), NCT00532233 and NCT01266135). However, these have not yet demonstrated efficacy in this setting [10-12].

SAR156597 is an engineered humanised bispecific IgG4 antibody that utilises an innovative tetravalent bispecific tandem immunoglobulin format to bind and neutralise circulating IL-4 and IL-13 [13]. A phase 1b/2a trial (NCT01529853) in patients with IPF found that SAR156597 reduced the level of thymus- and activation-regulated chemokine (TARC) [14], a protein biomarker directly induced by IL-4 and IL-13 [15].

We conducted a phase $2 \mathrm{~b}$ trial (NCT02345070) to evaluate the efficacy and safety of two dose levels/ regimens of SAR156597, in comparison with placebo, administered to patients with IPF over 52 weeks.

\section{Methods}

Patient population

The study included patients with IPF, both those with and without background anti-fibrotic therapy (pirfenidone or nintedanib). Key inclusion and exclusion criteria are outlined in table 1.

\section{Study design}

DRI11772 was a multinational randomised double-blind placebo-controlled phase $2 \mathrm{~b}$ trial, designed to evaluate the safety and efficacy of two dose levels/regimens of SAR156597 (200 mg once every week or once

\section{TABLE 1 Key inclusion and exclusion criteria}

\section{Inclusion criteria}

Adult male or female patients with documented diagnosis of IPF according to the following criteria:

Exclusion of other known causes of interstitial lung disease

(e.g. domestic and occupational environmental exposures, AND connective tissue disease and drug toxicity) ${ }^{\#}$

Combination of patterns of UIP on HRCT images of OR chest and on surgical lung biopsy (when obtained)

Presence of possible UIP pattern" on HRCT images of chest AND additional evidence of traction bronchiectasis as assessed by an experienced chest radiologist (central review $w^{\text {I] }}$

Signed written informed consent

\section{Exclusion criteria}

Age $\leqslant 40$ years

IPF disease diagnosis $>5$ years

FVC $\%$ pred $<40 \%$

DLco corrected for haemoglobin \% pred $<30 \%$

Severe chronic obstructive bronchitis as characterised by $\mathrm{FEV}_{1} / \mathrm{FVC}<0.70$

Known diagnosis of significant respiratory disorders other than IPF Pulmonary artery hypertension requiring a specific treatment Currently listed and/or anticipated to be listed for lung transplantation within the next 6 months (on an active list) History of vasculitis or connective tissue disorders or antineutrophil cytoplasmic auto-antibody positive Acute myocardial infarction within 6 months prior to screening

IPF: idiopathic pulmonary fibrosis; UIP: usual interstitial pneumonia; HRCT: high-resolution computed tomography; FVC: forced vital capacity; DLCo: diffusing capacity of the lung for carbon monoxide; FEV1: forced expiratory volume in $1 \mathrm{~s}$. \#: as defined by 2011 American Thoracic Society/European Respiratory Society/Japanese Respiratory Society/Latin American Thoracic Association guidelines [1]; ๆ: the patterns of UIP on HRCT images of chest and histopathology in surgical lung biopsy (if conducted) were reviewed by central reviewers (a radiologist and a pathologist experienced in interstitial lung diseases) to confirm diagnosis of IPF for consistency. 
every 2 weeks) administered subcutaneously to patients with IPF over 52 weeks. Following screening (up to 4 weeks), patients were randomised with a balanced allocation ratio (1:1:1) to receive 1) SAR156597 once every week (QW arm); 2) SAR156597 once every 2 weeks, alternating with placebo administered subcutaneously once every 2 weeks (Q2W arm); or 3) placebo once every week (placebo arm) (figure 1).

The randomisation was stratified; patients were grouped into two strata according to background anti-fibrotic therapy (pirfenidone or nintedanib). A blocked randomisation schedule was generated with records pre-allocated to each stratum (two strata) and study arm (three arms) with a balanced allocation ratio (1:1:1).

The study was conducted according to the principles of the Declaration of Helsinki and all applicable amendments; the International Conference on Harmonisation of Technical Requirements for the Registration of Pharmaceuticals for Human Use guidelines for good clinical practice; and all applicable laws, rules and regulations. All participants provided written informed consent.

\section{Study procedures}

Pulmonary function tests (diffusing capacity of the lung for carbon monoxide (DLCO) and forced vital capacity (FVC) ) and pulse oximetry were performed at screening, baseline and weeks 12, 24, 36, 52 and 64. Chest high-resolution computed tomography (HRCT) was performed at baseline, if not performed within 1 year prior to screening, and at week 52. Blood samples for biomarker analysis were taken at baseline and weeks 24 and 52, and at baseline and weeks 4, 12, 24, 36, 52 and 64 for antidrug antibodies (ADA) and pharmacokinetic (PK) analyses. Safety was evaluated over 64 weeks.

\section{Study assessments}

The primary efficacy end-point was absolute change from baseline in FVC \% predicted at 52 weeks. Secondary efficacy end-points comprised disease progression (defined as a decrease in absolute FVC \% pred $\geqslant 10 \%$, a decrease in absolute DLCO $\%$ pred $\geqslant 15 \%$, lung transplant or death at 52 weeks) and all-cause mortality at 52 weeks. Exploratory efficacy end-points comprised acute exacerbations of IPF at 52 weeks, and respiratory and non-elective hospitalisations at 52 weeks. Acute exacerbations of IPF were confirmed by the local investigators who responded to a series of questions in line with the currently accepted definition [16].

Safety assessments comprised adverse events (AEs) and treatment-emergent AEs (TEAEs); physical examination and body weight; vital signs and 12-lead ECG; clinical laboratory evaluations; and tolerability at the investigational medicinal product (IMP) injection site.

Other assessments included biomarker quantification (TARC and periostin), immunogenicity testing for $\mathrm{ADA}$ and $\mathrm{PK}$ analysis.

\section{Statistical analyses}

It was estimated that the SD of the absolute change in FVC \% pred at 52 weeks would be $\sim 12 \%$. For the primary efficacy end-point, 92 patients per treatment group would yield $80 \%$ power to detect a $5 \%$ difference between the treatment groups and placebo. To allow for dropouts, $\sim 100$ patients were randomised into each treatment group. Sample size calculation did not take background therapy into account.

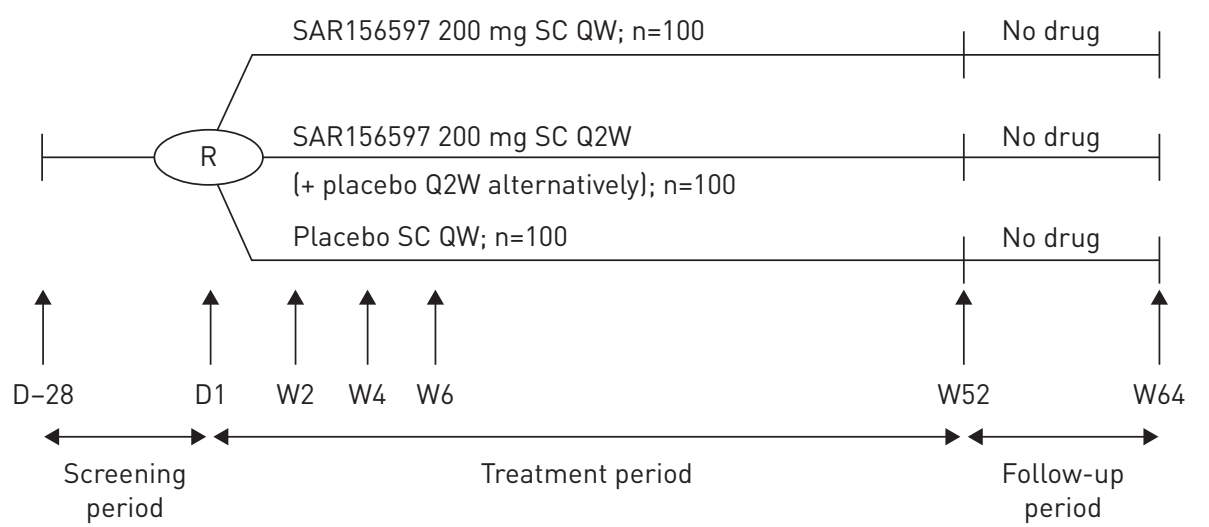

FIGURE 1 Study design. R: randomisation; SC: subcutaneous; QW: once every week; Q2W: once every 2 weeks; D: day; W: week. 


\section{Analysis populations}

The modified intention-to-treat population (primary population for efficacy analyses) included all patients who received at least one injection of IMP and had a valid baseline and at least one post-baseline FVC \% pred measurement. The safety population included all randomised patients who received at least one injection of IMP. PK and ADA analyses were conducted for all randomised and treated patients who had provided at least one blood sample.

The primary efficacy analysis comprised a rank-based analysis of covariance (RANCOVA), combining both continuous data (change from baseline in FVC \% pred) and clinical events (death and transplantation) to take into account informative missing values for FVC \% pred in case of death or lung transplantation. For each dose level/regimen of SAR156597, all participating patients (regardless of treatment assignment) were ranked according to a pre-specified hierarchical ranking of outcomes. If a comparison of deaths could not be conducted, then a comparison of lung transplantation was conducted; if this could not be conducted, then a comparison was conducted of change from baseline in FVC \% pred at the latest time point at which data from both comparators were available. The RANCOVA model was adjusted on the stratification factor (based on background anti-fibrotic therapy) and included treatment group as the main factor and baseline FVC \% pred as a continuous covariate.

Secondary time-to-event analyses used the log-rank test and Cox proportional hazards regression model, and results are presented as Kaplan-Meier curves. Safety, PK and biomarker variables were analysed using descriptive statistics. Changes in biomarker levels were analysed using a mixed model for repeated measures.

\section{Results}

Patient disposition

A total of 652 patients were assessed for eligibility, of which 327 patients were randomised; 325 received treatment with placebo ( $\mathrm{n}=109)$, SAR156597 Q2W ( $\mathrm{n}=108)$ or SAR156597 QW ( $\mathrm{n}=108)$ (figure 2). Overall, 167 patients $(51.1 \%)$ were receiving background therapy (pirfenidone or nintedanib) at study entry. In all, 253 patients $(77.4 \%)$ completed the study treatment period. The primary and safety populations each included 325 patients.

There were 320 patients (49\%) who failed screening in this trial; the most common reasons for screen failure were a positive result on a QuantiFERON-TB Gold test at screening $(n=114,36 \%)$, not meeting the inclusion criteria of a central review of HRCT and lung biopsies $(n=67,21 \%)$, and a history of vasculitis or connective tissue disorders or testing positive for antineutrophil cytoplasmic antibodies $(n=33,10 \%)$.

\section{Patient characteristics}

Patient demographics and baseline characteristics were similar across treatment arms (table 2). Patients' IPF characteristics at baseline were comparable to those of other IPF cohorts [17-19]. Patients who received background therapy generally were diagnosed with IPF earlier and had an earlier onset of first IPF symptom compared with those without background therapy (supplementary table S1).

\section{Efficacy}

There was no trend or significant difference in the primary end-point between the SAR156597 treatment arms and placebo (table 3; figure 3a). With RANCOVA, incidences of clinical events in the placebo arm were $9.2 \%$ for death and $0 \%$ for lung transplantation, with a non-imputed (for death and lung transplantation) mean change from baseline in FVC \% pred at 52 weeks of $-4.4 \%$; for the Q2W arm, the corresponding incidence rates were $6.5 \%, 0.9 \%$ and $-4.7 \%$; for the QW arm, they were $9.3 \%, 2.8 \%$ and $-4.9 \%$ (Q2W versus placebo, $\mathrm{p}=0.42$; QW versus placebo, $\mathrm{p}=0.39$ ). With imputation for death, lung transplant and other missing data, the least squares (LS) mean change from baseline in FVC \% pred at 52 weeks was $-5.8 \%,-5.2 \%$ and $-6.3 \%$ for the placebo, Q2W and QW arms, respectively (Q2W versus placebo, $\mathrm{p}=0.59$; $\mathrm{QW}$ versus placebo, $\mathrm{p}=0.63$ ).

There were also no differences between treatment arms when data were stratified by background therapy: mean change from baseline in FVC \% pred at 52 weeks was $-3.7 \%,-5.2 \%$ and $-4.8 \%$ for the placebo, Q2W and QW arms, respectively, in patients without background therapy, and $-5.1 \%,-4.2 \%$ and $-5.0 \%$, respectively, in those with background therapy.

\section{Secondary end-points}

No significant difference or trend was observed in disease progression between treatment groups over 52 weeks (Q2W versus placebo, $\mathrm{p}=0.90$; $\mathrm{QW}$ versus placebo, $\mathrm{p}=0.31$ ) (table 3 ; figure $3 \mathrm{~b}$ ). Similarly, no significant difference or trend was observed in all-cause mortality between treatment groups over 52 weeks (figure 3c). There were 10, 8 and 14 deaths in the placebo, Q2W and QW treatment arms, respectively (associated probability of all-cause mortality was $0.09,0.08$ and 0.13 , respectively). 


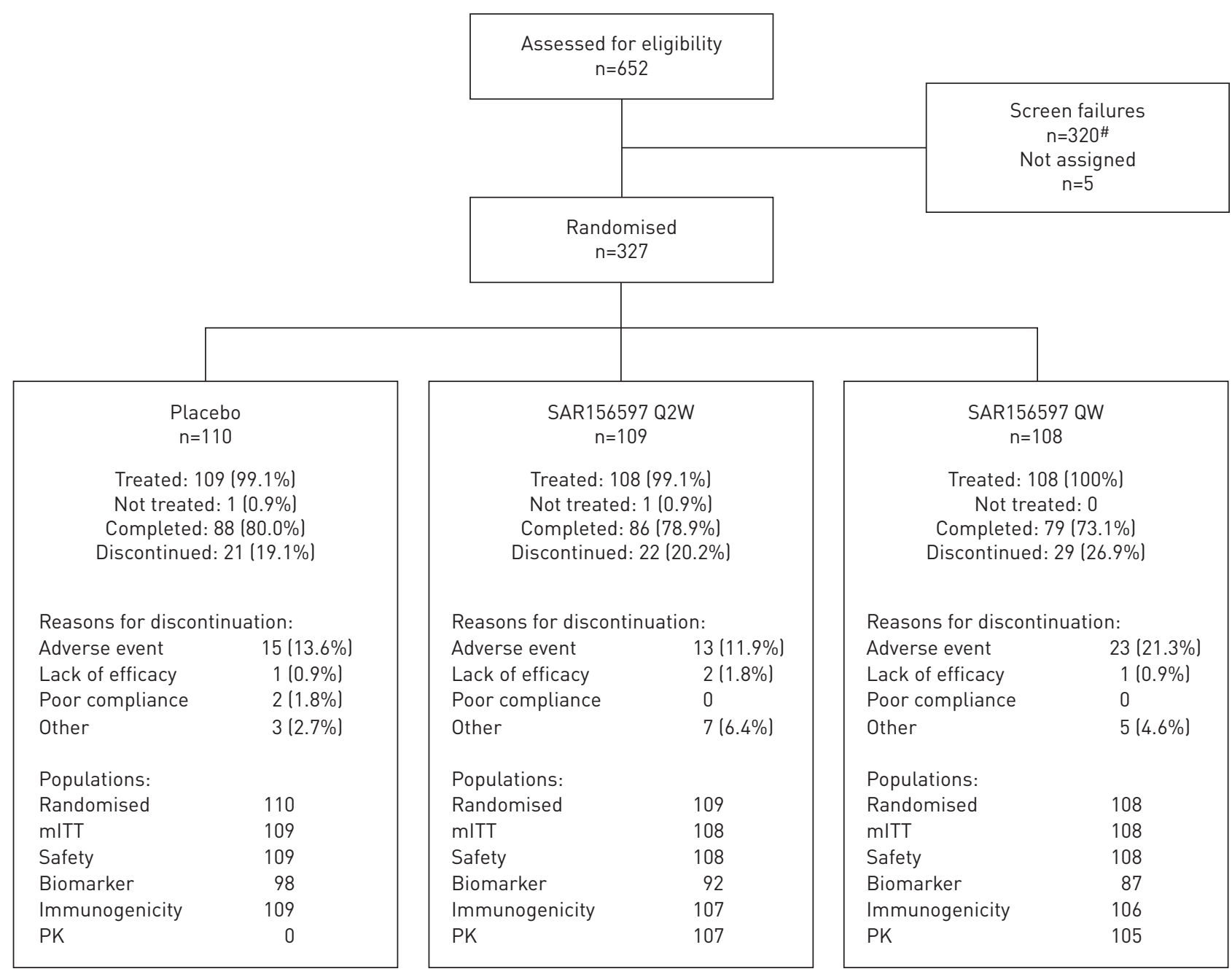

FIGURE 2 Patient disposition. Q2W: once every 2 weeks; QW: once every week; mITT: modified intention-to-treat; PK: pharmacokinetic. \#: 320 patients (49\%) were screen failures in this trial; the most common reasons were positive QuantiFERON-TB Gold test at screening (n=114; $36 \%$ ), patients did not meet the inclusion criteria of central review of high-resolution computed tomography and lung biopsies ( $=67 ; 21 \%$ ), and a history of vasculitis or connective tissue disorders or positive antineutrophil cytoplasmic antibodies ( $n=33 ; 10 \%)$. A patient could have more than one criterion not met.

\section{Exploratory end-points}

There were fewer cases of acute exacerbations of IPF in the SAR156597 treatment arms (Q2W: $\mathrm{n}=5$, 4.6\%; QW: $n=7,6.5 \%)$ than the placebo arm (n=9, 8.3\%) (table 3). More patients were hospitalised for any cause and for respiratory causes in the QW arm (any cause: $\mathrm{n}=38,35.2 \%$; respiratory causes: $\mathrm{n}=24$, 22.2\%) compared with the Q2W $(n=22,20.4 \% ; n=13,12.0 \%)$ and placebo $(n=18,16.5 \% ; n=15,13.8 \%)$ arms (table 3).

\section{Biomarker data}

There was a significant decrease in circulating TARC level in patients treated with SAR156597 compared to placebo, confirming target engagement. At 52 weeks, the LS mean change from baseline in TARC was $+118.1 \mathrm{ng} \cdot \mathrm{L}^{-1}(+27.6 \%),-6.8 \mathrm{ng} \cdot \mathrm{L}^{-1}(-4.4 \%)$ and $-82.0 \mathrm{ng} \cdot \mathrm{L}^{-1}(-12.4 \%)$ for the placebo, Q2W and QW arms, respectively (Q2W versus placebo, $\mathrm{p}=0.04$; $\mathrm{QW}$ versus placebo, $\mathrm{p}=0.002$ ). In patients without background therapy, the LS mean change from baseline in TARC at 52 weeks was $+132.4 \mathrm{ng} \cdot \mathrm{L}^{-1}(+35.6 \%)$, $-76.9 \mathrm{ng} \cdot \mathrm{L}^{-1}(-1.2 \%)$ and $-147.3 \mathrm{ng} \cdot \mathrm{L}^{-1}(-10.9 \%)$ for the placebo, Q2W and QW arms, respectively (Q2W versus placebo, $\mathrm{p}=0.02$; $\mathrm{QW}$ versus placebo, $\mathrm{p}=0.003)$; in those with background therapy, it was $+108.3 \mathrm{ng} \cdot \mathrm{L}^{-1} \quad(+58.0 \%),+74.0 \mathrm{ng} \cdot \mathrm{L}^{-1}(+28.7 \%)$ and $-28.4 \mathrm{ng} \cdot \mathrm{L}^{-1}(-9.1 \%)$, respectively (Q2W versus placebo, $\mathrm{p}=0.69$; $\mathrm{QW}$ versus placebo, $\mathrm{p}=0.11$ ).

There were no significant differences between treatment arms in changes from baseline to week 52 in levels of periostin $(+4.7 \%,+2.8 \%$ and $+0.4 \%$ in the placebo, Q2W and QW arms, respectively). 
TABLE 2 Patient demographics and baseline characteristics (randomised population)

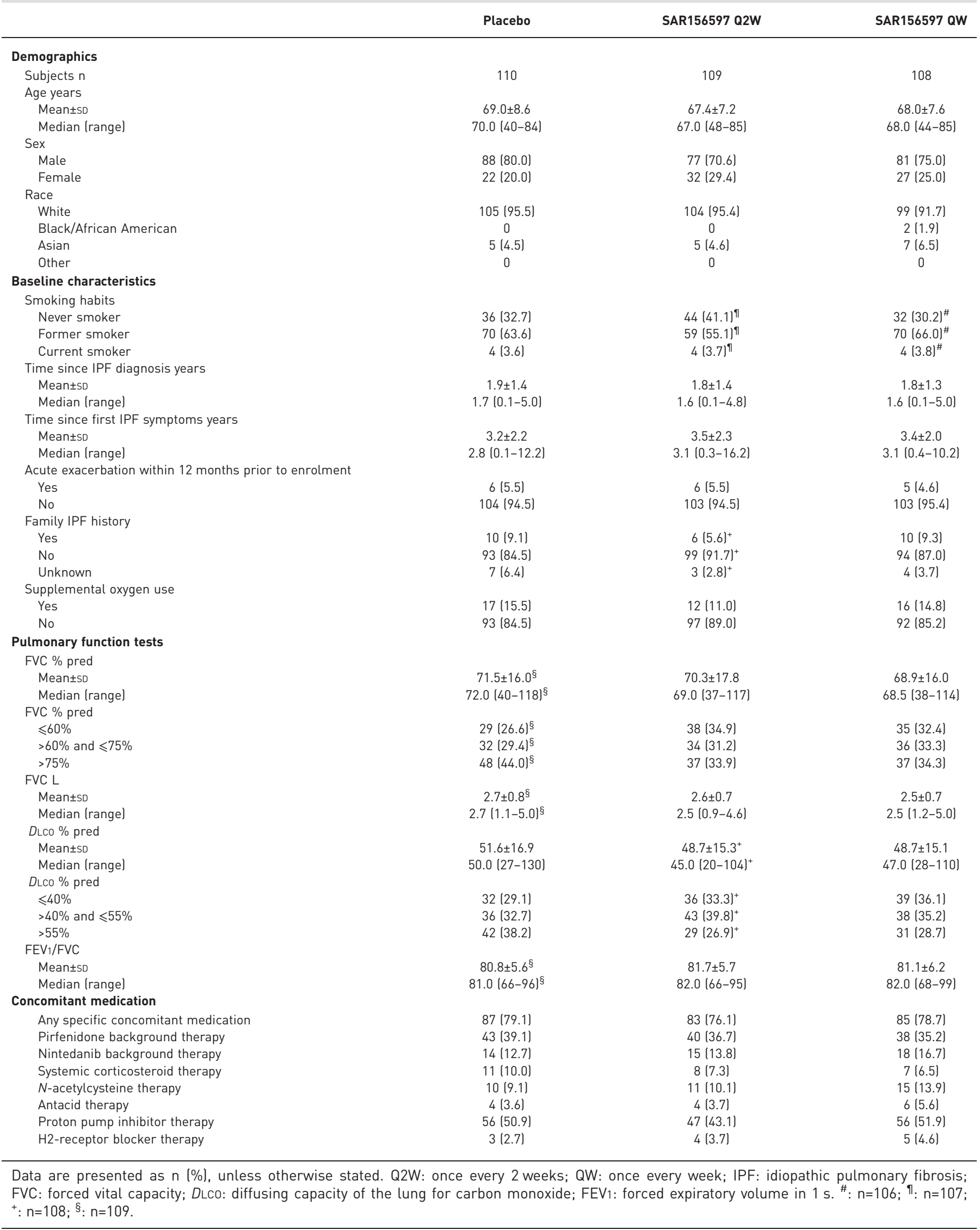


TABLE 3 Summary of primary, secondary and exploratory efficacy end-points (modified intention-to-treat population)

\begin{tabular}{|c|c|c|c|}
\hline & Placebo & SAR156597 Q2W & SAR156597 QW \\
\hline Subjects $\mathrm{n}$ & 109 & 108 & 108 \\
\hline \multicolumn{4}{|l|}{ Primary end-point } \\
\hline Death $\mathrm{n}(\%)$ & $10(9.2)$ & $7(6.5)$ & 10 (9.3) \\
\hline Lung transplant $\mathrm{n}(\%)$ & 0 & $1(0.9)$ & $3(2.8)$ \\
\hline \multicolumn{4}{|c|}{ Change from baseline to 52 weeks in FVC \% pred } \\
\hline \multicolumn{4}{|c|}{ Sensitivity analysis } \\
\hline \multicolumn{4}{|c|}{ Change from baseline to 52 weeks in FVC \% pred } \\
\hline LS mean \pm SE & $-5.8 \pm 0.7$ & $-5.2 \pm 0.7$ & $-6.3 \pm 0.8$ \\
\hline LS mean difference versus placebo \pm SE & & $0.6 \pm 1.0$ & $-0.5 \pm 1.1$ \\
\hline $95 \% \mathrm{Cl}$ & & $-1.5-2.6$ & $-2.6-1.6$ \\
\hline p-value versus placebo & & 0.59 & 0.63 \\
\hline HR $(95 \%$ Cl) versus placebo & & $1.023(0.640-1.636)$ & $1.255(0.801-1.966)$ \\
\hline $\mathrm{p}$-value versus placebo & & 0.90 & 0.31 \\
\hline \multicolumn{4}{|c|}{ Decomposition of the disease progression composite end-point } \\
\hline \multicolumn{4}{|c|}{ Absolute decrease in FVC $\%$ pred $\geqslant 10$} \\
\hline Number of events & 14 & 13 & 20 \\
\hline Probability of events & 0.158 & 0.262 & 0.389 \\
\hline HR versus placebo & & 0.955 & 1.501 \\
\hline \multicolumn{4}{|l|}{ Absolute decrease in $D$ Lco $\%$ pred $\geqslant 15$} \\
\hline Number of events & 13 & 15 & 12 \\
\hline Probability of events & 0.362 & 0.206 & 0.138 \\
\hline HR versus placebo & & 1.197 & 0.981 \\
\hline \multicolumn{4}{|l|}{ Lung transplant } \\
\hline Number of events & 0 & 1 & 2 \\
\hline Confirmed acute IPF exacerbation & 9 (8.3) & $5(4.6)$ & $7(6.5)$ \\
\hline \multicolumn{4}{|l|}{ Respiratory and non-elective hospitalisations } \\
\hline Any hospitalisation & $18(16.5)$ & $22(20.4)$ & $38(35.2)$ \\
\hline \multicolumn{4}{|l|}{ Type of hospitalisation } \\
\hline Elective & $3(2.8)$ & 9 (8.3) & 10 (9.3) \\
\hline Non-elective & $16(14.7)$ & $17(15.7)$ & $32(29.6)$ \\
\hline \multicolumn{4}{|l|}{ Reason for hospitalisation } \\
\hline Respiratory & 15 (13.8) & $13(12.0)$ & $24(22.2)$ \\
\hline Other & $5(4.6)$ & $12(11.1)$ & $22(20.4)$ \\
\hline
\end{tabular}

Q2W: once every 2 weeks; QW: once every week; RANCOVA: rank-based analysis of covariance; FVC: forced vital capacity; LS: least squares; HR: hazard ratio; DLCO: diffusing capacity of the lung for carbon monoxide; IPF: idiopathic pulmonary fibrosis; TEAE: treatment-emergent adverse event. \#: RANCOVA analysis is based on a global ranking combining both clinical events (death and transplantation) and continuous data (change from baseline in FVC \% pred). See Methods section for more detail.

\section{Immunogenicity}

SAR156597 was associated with low-level immunogenicity. In the placebo group, two of 109 patients were ADA positive at baseline and remained so until week $24(n=1)$ and week $64(n=1)$. In the Q2W arm, all patients were ADA negative at baseline; one of 107 patients developed transient positivity of ADA at week 36 only. In the QW arm, all 106 patients were ADA negative at baseline and throughout the study. 

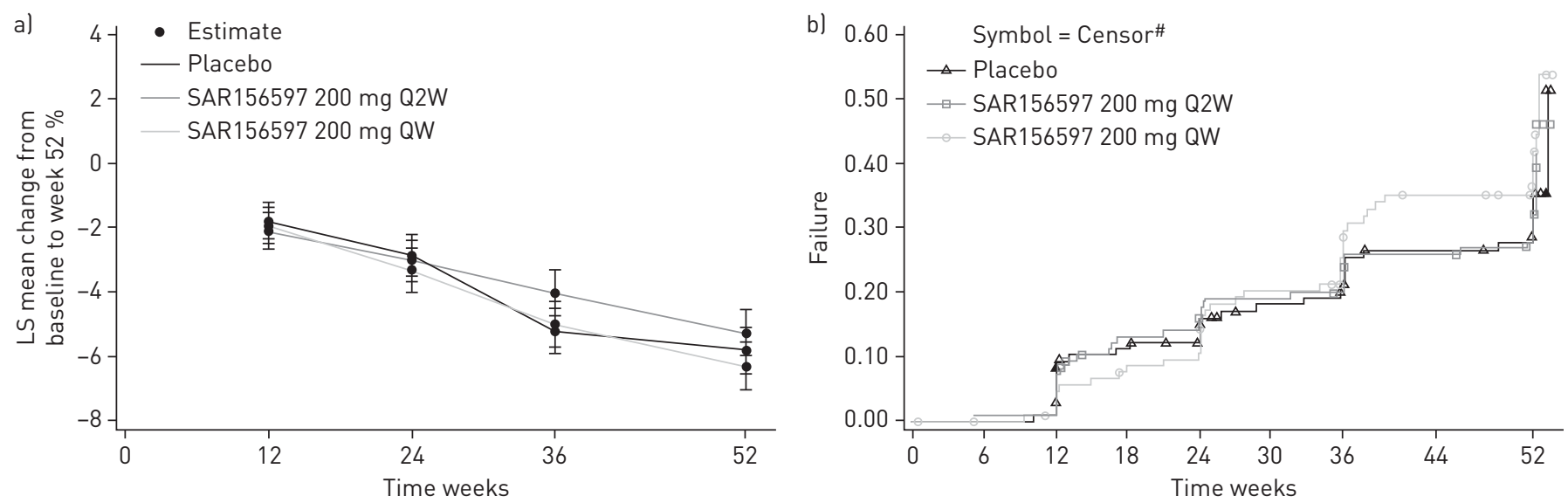

Number at risk

Placebo

$\begin{array}{rrrrrrrrr}109 & 109 & 108 & 94 & 90 & 80 & 77 & 68 & 61 \\ \text { SAR 200 mg Q2W } & & & & & & & \\ 108 \quad 107 & 107 & 91 & 90 & 84 & 82 & 71 & 57 \\ \text { SAR 200 mg QW } & & & & & & & \\ 108 \quad 106 & 104 & 96 & 94 & 81 & 75 & 58 & 53\end{array}$
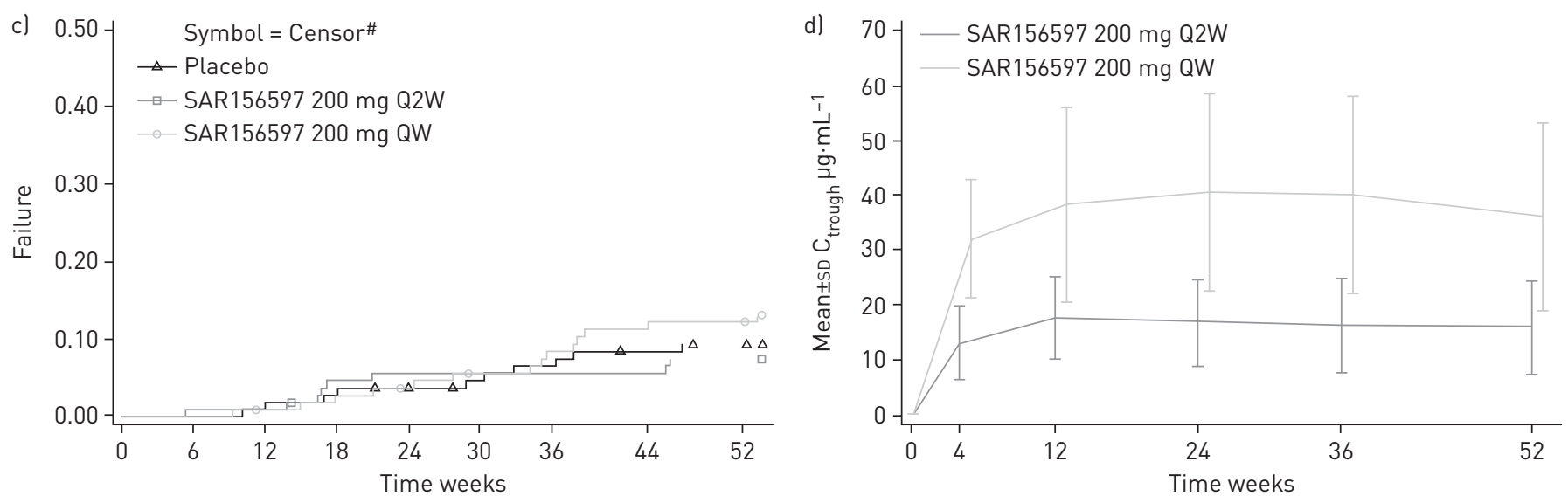

Number at risk

\begin{tabular}{|c|c|c|c|c|c|c|c|c|}
\hline \multicolumn{9}{|l|}{ Placebo } \\
\hline 109 & 109 & 108 & 106 & 104 & 101 & 99 & 96 & 94 \\
\hline \multicolumn{9}{|c|}{ SAR 200 mg Q2W } \\
\hline 108 & 107 & 107 & 102 & 101 & 101 & 101 & 101 & 99 \\
\hline \multicolumn{9}{|c|}{ SAR 200 mg QW } \\
\hline 108 & 108 & 106 & 105 & 102 & 99 & 96 & 93 & 92 \\
\hline
\end{tabular}

FIGURE 3 a) Absolute change from baseline in forced vital capacity (FVC) \% pred: adjusted mean \pm SE profile over time by treatment group (modified intention-to-treat (mITT) population). b) Disease progression: Kaplan-Meier cumulative incidence curve by treatment group (mITT population). Disease progression was defined as a decrease in absolute FVC $\%$ pred $\geqslant 10 \%$ or a decrease in absolute diffusing capacity of the lung for carbon monoxide (DLCo) \% pred $\geqslant 15 \%$ or lung transplant or death at 52 weeks. c) All-cause mortality: Kaplan-Meier cumulative incidence curve by treatment group (mITT population). d) Pharmacokinetic (PK) analysis: mean \pm SD lowest concentration the drug reached before next dose was administered ( $C_{\text {trough }}$ ) profiles over time by treatment group (PK population). Q2W: once every 2 weeks; $Q$ W: once every week. \#: patients not meeting the definition for disease progression (defined as a decease in absolute FVC $\%$ pred $\geqslant 10 \%$, a decrease in absolute DLCo $\%$ pred $\geqslant 15 \%$, lung transplant or death at 52 weeks) were considered censored at the time of their week 52 assessment but not later than day 375 . For patients with a missing assessment at week 52 , the censoring time was considered to be the time of the last measured assessment.

\section{PK analysis}

For both the Q2W and QW treatment arms, steady state was reached by week 12. The lowest concentration SAR156597 reached before the next dose $\left(\mathrm{C}_{\text {trough }}\right)$ increased by 2.3 -fold at week 52 between the QW and Q2W dosing regimens, consistent with an expected two-fold increase (figure 3d). No difference in SAR156597 $\mathrm{C}_{\text {trough }}$ concentrations was observed in patients treated with or without background anti-fibrotic therapy prior to randomisation. 
TABLE 4 Summary of TEAEs (safety population)

Placebo SAR156597 Q2W SAR156597 QW

\begin{tabular}{|c|c|c|c|}
\hline Patients n & 109 & 108 & 108 \\
\hline Patients with any TEAE & 99 (90.8) & $102(94.4)$ & $100(92.6)$ \\
\hline \multicolumn{4}{|l|}{ Most frequently reported TEAEs ${ }^{\#}$} \\
\hline IPF & $19(17.4)$ & $19(17.6)$ & $30(27.8)$ \\
\hline Cough & $12(11.0)$ & $22(20.4)$ & $18(16.7)$ \\
\hline Diarrhoea & $16(14.7)$ & $23(21.3)$ & $12(11.1)$ \\
\hline Viral upper RTI & $16(14.7)$ & $18(16.7)$ & $12(11.1)$ \\
\hline Bronchitis & $13(11.9)$ & $18(16.7)$ & $11(10.2)$ \\
\hline \multicolumn{4}{|l|}{ Patients with TEAEs by intensity } \\
\hline Mild & 85 (78.0) & $83(76.9)$ & 85 (78.7) \\
\hline Moderate & $70(64.2)$ & $71(65.7)$ & $76(70.4)$ \\
\hline Severe & $22(20.2)$ & $24(22.2)$ & 42 (38.9) \\
\hline Patients with any treatment-related TEAE & $35(32.1)$ & $32(29.6)$ & $35(32.4)$ \\
\hline Patients with any SAE & $26(23.9)$ & $27(25.0)$ & $46(42.6)$ \\
\hline \multicolumn{4}{|l|}{ Most frequently reported SAEs" } \\
\hline IPF & $10(9.2)$ & 9 (8.3) & $22(20.4)$ \\
\hline Pneumonia & $4(3.7)$ & $3(2.8)$ & $8(7.4)$ \\
\hline Pulmonary hypertension & 0 & $2(1.9)$ & $2(1.9)$ \\
\hline Bronchitis & $1(0.9)$ & 0 & $2(1.9)$ \\
\hline Septic shock & $2(1.8)$ & 0 & $1(0.9)$ \\
\hline Pulmonary embolism & $2(1.8)$ & 0 & $1(0.9)$ \\
\hline Acute coronary syndrome & $1(0.9)$ & 0 & $2(1.9)$ \\
\hline Lung infection & 0 & $2(1.9)$ & 0 \\
\hline Respiratory failure & 0 & 0 & $2(1.9)$ \\
\hline Hepatic congestion & 0 & 0 & $2(1.9)$ \\
\hline Patients with any treatment-related SAE & $5(4.6)$ & $5(4.6)$ & $3(2.8)$ \\
\hline Patients with any TEAE leading to death & $11(10.1)$ & $6(5.6)$ & $13(12.0)$ \\
\hline \multicolumn{4}{|l|}{ Most frequently reported TEAEs leading to death" } \\
\hline IPF & $6(5.5)$ & 0 & $7(6.5)$ \\
\hline Pneumonia & $1(0.9)$ & $2(1.9)$ & 0 \\
\hline Septic shock & $2(1.8)$ & 0 & $1(0.9)$ \\
\hline Patients with any TEAE leading to discontinuation & 15 (13.8) & $13(12.0)$ & $23(21.3)$ \\
\hline \multicolumn{4}{|c|}{ Most frequently reported TEAEs leading to discontinuation" } \\
\hline IPF & 8 (7.3) & $4(3.7)$ & 9 (8.3) \\
\hline Pulmonary hypertension & 0 & $2(1.9)$ & 0 \\
\hline Transient ischaemic attack & 0 & $2(1.9)$ & 0 \\
\hline
\end{tabular}

Data presented as $n(\%)$, unless otherwise stated. TEAE: treatment-emergent adverse event; Q2W: once every 2 weeks; QW: once every week; IPF: idiopathic pulmonary fibrosis; RTI: respiratory tract infection; $\mathrm{SAE}$ : serious adverse event. ${ }^{\#}: \geqslant 15 \%$ patients in any arm; ${ }^{\uparrow}:>1$ patient in any arm.

\section{Safety}

TEAEs were observed in $90.8 \%, 94.4 \%$ and $92.6 \%$ of patients in the placebo, Q2W and QW arms, respectively (table 4). The most frequently reported TEAEs $(\geqslant 15 \%$ of patients in any arm) were worsening of IPF, cough, diarrhoea, viral upper respiratory tract infection and bronchitis. The TEAEs categorised by System Organ Class were generally balanced across the treatment arms, with the exception of cardiac disorders, which were reported more frequently in the QW arm (16.7\%) than in the Q2W (9.3\%) and placebo (6.4\%) arms. However, no individual cardiac disorder was reported in $\geqslant 5 \%$ of the patients in any arm.

The incidences of serious AEs (SAEs) and TEAEs leading to discontinuation were higher in the QW arm than in the Q2W and placebo arms. This was mainly driven by a higher incidence of worsening IPF and cardiac disorders in the QW arm, compared with the other arms. The only cardiac disorder reported as an SAE for more than one patient per arm was acute coronary syndrome, reported for two patients in the QW arm. A total of 30 patients (9.2\%) developed a TEAE that led to death (placebo: $\mathrm{n}=11,10.1 \%$; Q2W: $n=6,5.6 \%$; QW: $n=13,12.0 \%$ ). The most frequently reported TEAE leading to death was related to IPF.

The incidences of TEAEs, TEAEs considered related to the study drug and SAEs were higher in patients who received background anti-fibrotic therapy prior to randomisation than in those who did not (supplementary table S2). 
Increased alanine aminotransferase (defined as $\geqslant 3 \times$ upper limit of normal (ULN) if baseline level was $<\mathrm{ULN}$ or $\geqslant 2 \times \mathrm{ULN}$ if baseline level was $\geqslant \mathrm{ULN}$ ) was reported as an AE of special interest in nine patients, with the majority in the QW arm (placebo: $n=1,0.9 \%$; Q2W: $n=2,1.9 \%$; QW: $n=6,5.6 \%$ ). Weight decrease ( $\geqslant 5 \%$ from baseline) occurred more frequently in the Q2W (32.4\%) and QW (35.2\%) arms than in the placebo arm (23.9\%). There were no meaningful differences between treatment arms for other clinical laboratory parameters, vital signs, or findings from physical examination or ECG.

\section{Discussion}

In this phase 2 study, SAR156597 failed to demonstrate benefit in treating patients with IPF. The primary efficacy end-point (FVC change at 52 weeks) and key secondary efficacy end-points (disease progression and all-cause mortality) did not show a statistically significant difference between either of the SAR156597 treatment regimens and placebo, regardless of stratification based on background anti-fibrotic therapy. Results for the exploratory efficacy end-points were mixed. The occurrence of (investigator-reported) acute IPF exacerbations was relatively balanced between treatment arms, although there was a trend towards a decrease in acute IPF exacerbations in the SAR156597 arms compared with placebo. Respiratory and non-elective hospitalisations were, however, more frequently observed in the QW arm than the Q2W and placebo arms.

Although the incidence of TEAEs was similar across treatment arms, SAEs and TEAEs leading to discontinuation were more frequently observed in the QW arm than in the Q2W and placebo arms. This imbalance in TEAEs was mainly driven by a higher incidence of IPF events (progression or acute exacerbation) and cardiac events in the QW arm compared with the other arms. The IPF events may have contributed to the imbalance in cardiac events because they frequently occurred contemporaneously. In other respects, the safety profile observed in the three treatment arms was generally similar. SAR156597 was associated with a low level of immunogenicity in this study.

TARC is directly induced by IL-4 and IL-13 receptor activation [15] and is one of the key biomarkers of target engagement by SAR156597. In our study, SAR156597 reduced TARC levels in an apparently dose-dependent way compared to placebo. These findings are consistent with those of a previous phase 1b/2a trial (NCT01529853) and indicate that SAR156597 engaged with its target. However, this did not result in any discernible benefits in terms of IPF treatment, suggesting that IL-4 and IL-13 may not play as dominant a role in disease progression and pathogenesis as previously thought. Given the trend towards decreased occurrence of acute IPF exacerbations in the SAR156597 arms compared with placebo, it may be speculated that different pathways exist for IPF exacerbations versus lung function. Recently, inhibition of the IL-13 pathway with tralokinumab, a human anti-IL-13 monoclonal antibody, showed no effect on the rate of decline of lung function over 52 weeks in a phase 2 trial in patients with mild-to-moderate IPF [10]. In that trial, tralokinumab-treated patients had a numerically greater decline in FVC than placebo-treated patients, and the percentage of subjects with a decline in FVC $\%$ pred $\geqslant 10 \%$ at week 52 was greater for patients treated with tralokinumab than with placebo [10]. In another recent phase 2 trial, the addition of lebrikizumab to background pirfenidone therapy was similarly not associated with a treatment benefit in terms of FVC change over 52 weeks; however, as in our trial, there was a suggestion of benefit in terms of acute IPF exacerbations, as well as mortality [12]. These data question the role of IL-13 in IPF pathophysiology. Thus, although the present study failed to demonstrate benefit for SAR156597 in IPF treatment, it is nonetheless a valid negative study.

Approximately half the patients included in this study were receiving background anti-fibrotic therapy before randomisation. Such patients are likely to have more severe disease than those naive to anti-fibrotic treatment, which may have introduced bias into the study design, and sub-analysis of the primary efficacy end-point results revealed no trends or differences between treatment arms when data were stratified by background therapy. The incidences of TEAEs, treatment-related TEAEs and SAEs were higher in patients receiving background anti-fibrotic therapy than in those who were not. The most commonly reported TEAEs and SAEs were IPF events (progression or acute exacerbation), perhaps supporting the idea that patients who were receiving background anti-fibrotic therapy had more severe disease than those naïve to this therapy. There is no known reason to suggest that drug-drug interactions between SAR156597 and background anti-fibrotic therapy may have affected the observed safety findings, although this was not specifically analysed in the study. No difference in $\mathrm{C}_{\text {trough }}$ was observed between patients who were and those who were not receiving background anti-fibrotic therapy; however, the sample size calculation did not take into account background anti-fibrotic therapy use. These findings may have implications for the methodology employed for future IPF trials.

In the present trial, screen failure rate was $49 \%$. One of the main reasons for screen failure was a positive QuantiFERON-TB Gold test, accounting for 36\% of these cases. The high screen failure rate is in line with 
published reports of other IPF clinical trials, such as the INPULSIS, CAPACITY, ASCEND, PANTHER IPF, ARTEMIS IPF and RAINIER IPF trials [17-22].

In summary, this valid negative study failed to demonstrate benefit for SAR156597 in the treatment of IPF. The SAR156597 200-mg QW dose was associated with a more unfavourable safety profile than the SAR156597 200-mg Q2W dose and placebo. While neutralisation of both IL-4 and IL-13 by humanised bispecific IgG4 antibody did not have a successful outcome on FVC and other relevant clinical events in patients with IPF, further research is required to determine whether IL-4 and IL-13 are involved in IPF exacerbations versus lung function.

Acknowledgements: The authors thank all the principal investigators who participated in this study, namely: Gabriel Garcia, Ana Maria Stok, Carlos Elias, Gaston De Stefano, Patricia Malamud, Alberto Tolcachier, Daniel Chambers, David Langton, Peter Bremner, Allan Glanville, Tamera Corte, Nasreen Khalil, Shane Shapera, Juan Carlos Rodriguez, Rafael Silva, Juana Paviè, Patricia Schonffeldt, Laura Mendoza, Manuel Barros, Carlos Matiz Bueno, Jaime Sanchez, Carlos Salgado, Martina Sterclova, Vit Havel, Jiri Votruba, Vitezslav Kolek, Saher Shaker, Elisabeth Bendstrup, Vincent Cottin, Benoit Wallaert, Sylvain Marchand-Adam, Charles-Hugo Marquette, Arnaud Bourdin, Hilario Nunes, Gregoire Prevot, Pascal Chanez, Michael Kreuter, Michael Pfeifer, Dirk Koschel, Andreas Günther, Antje Prasse, Katerina Antoniou, Yochai Adir, Mordechai Kramer, Michael Segel, David Shitrit, Gershon Fink, Venerino Poletti, Sara Tomassetti, Carlo Albera, Carlo Vancheri, Paola Rottoli, Sergio Harari, Pierluigi Paggiaro, Hwan Jeong Sung, Joo Lee Eun, Ho In Kwang, Whan Kim Young, Woo Song Jin, Sik Park Choon, Sun Park Jong, Roberto Mercado Longoria, Alejandra Ramírez Venegas, Alberto Rosas Mario, Rodolfo Posadas, Antonio Morais, Alexandra Neves, Maria Molina, Jacobo Sellarés, Ana Villar, Alberto Cano Jiménez Esteban, Belén Núñez, Christian García Fadul, Amalia Moreno Gomez De Bonilla, Esin Tuncay, Nesrin Mogulkoc, Hatice Turker, Gulfer Okumus, Ozlem Ozdemir Kumbasar, Gul Ongen, Toby Maher, Helen Parfrey, Michael Gibbons, Felix Woodhead, Richard Enelow, Robert Sussman, Mark Gotfried, James Utz, Mark Pollock, Rade Tomic, David Winslow, Craig Glazer, Rany Condos, Paul Strachan, Maryl Kreider, Craig Thurm, Srihari Veeraraghavan, Abubakr Bajwa, Lisa Jones, Neal Warshoff, Jean-Claude Labissiere, Jonathan Ilowite, Tomasz Ziedalski and Neil Ettinger, as well as all of their co-investigators. The authors also give special thanks to David Hansell and Andrew Nicholson for their review of all high-resolution computed tomography chest scans and histology of lung biopsies to ensure accurate diagnosis of IPF at study entry. Writing support was provided by Sarah J. Petit (PAREXEL, London, UK), with funding from Sanofi-Aventis R\&D.

Author contributions: All authors take responsibility for (are the guarantor of) the accuracy and integrity of the content of this manuscript. All authors contributed to the data analysis and interpretation of the results and writing or critical reviewing of the manuscript, as well as providing final approvals. G. Raghu, L. Richeldi, B. Crestani, R. Bejuit, C. Antoni and C. Soubrane were involved in the concept and study design; P. Wung, R. Bejuit and C. Soubrane participated in the data acquisition.

Conflict of interest: G. Raghu reports consultant and advisor fees from Sanofi during the conduct of the study, and consultant and advisor fees from Bellerophan, Boehringer Ingelheim, BMS, FibroGen, Gilead, Nitto, Parata, Promedior and Veracyte, outside the submitted work. L. Richeldi reports grants and personal fees from InterMune, personal fees (for advisory board membership) from Medimmune, Roche and Takeda, personal fees (for consultancy) from Biogen, Sanofi-Aventis, ImmuneWorks and Pliants Therapeutics, speakers fees from Shionogi and Cipla, and fees from Boehringer Ingelheim as a member of a steering committee, outside the submitted work. B. Crestani reports personal fees from Sanofi, grants, personal fees and non-financial support (for speaking, travel and research) from Boehringer Ingelheim and Roche, grants for research from CARDIF and LVL, personal fees and non-financial support (for speaking and travel) from AstraZeneca, and grants and non-financial support (for research and travel) from Medlmmune, outside the submitted work. P. Wung has nothing to disclose. R. Bejuit reports personal fees (salary) from Sanofi, during the conduct of the study and outside the submitted work. C. Esperet reports personal fees (salary) from Sanofi, during the conduct of the study and outside the submitted work. C. Antoni reports personal fees (salary) from Sanofi, during the conduct of the study and outside the submitted work. C. Soubrane reports personal fees (salary) from Sanofi, during the conduct of the study and outside the submitted work.

Support statement: This study was funded by Sanofi-Aventis R\&D. Funding information for this article has been deposited with the Crossref Funder Registry.

\section{References}

1 Raghu G, Collard HR, Egan JJ, et al. An official ATS/ERS/JRS/ALAT statement: idiopathic pulmonary fibrosis: evidence-based guidelines for diagnosis and management. Am J Respir Crit Care Med 2011; 183: 788-824.

2 Raghu G, Richeldi L. Current approaches to the management of idiopathic pulmonary fibrosis. Respir Med 2017; 129: $24-30$.

3 Marshall DC, Salciccioli JD, Shea BS, et al. Trends in mortality from idiopathic pulmonary fibrosis in the European Union: an observational study of the WHO mortality database from 2001-2013. Eur Respir J 2018; 51: 1701603.

4 Raghu G, Rochwerg B, Zhang Y, et al. An Official ATS/ERS/JRS/ALAT Clinical Practice Guideline: Treatment of idiopathic pulmonary fibrosis. An update of the 2011 Clinical Practice Guideline. Am J Respir Crit Care Med 2015; 192: e3-e19.

5 Park SW, Ahn MH, Jang HK, et al. Interleukin-13 and its receptors in idiopathic interstitial pneumonia: clinical implications for lung function. J Korean Med Sci 2009; 24: 614-620.

6 Hancock A, Armstrong L, Gama R, et al. Production of interleukin 13 by alveolar macrophages from normal and fibrotic lung. Am J Respir Cell Mol Biol 1998; 18: 60-65.

7 Jakubzick C, Choi ES, Joshi BH, et al. Therapeutic attenuation of pulmonary fibrosis via targeting of IL-4- and IL-13-responsive cells. J Immunol 2003; 171: 2684-2693. 

fibrosis. I Thorac Dis 2013; 5: 48-73.

9 Izuhara K, Conway SJ, Moore BB, et al. Roles of periostin in respiratory disorders. Am J Respir Crit Care Med 2016; 193: 949-956.

10 Parker JM, Glaspole IN, Lancaster LH, et al. A phase 2 randomized controlled study of tralokinumab in subjects with idiopathic pulmonary fibrosis. Am J Respir Crit Care Med 2018; 197: 94-103.

11 Swigris JJ, Ogura T, Scholand M, et al. The RIFF study (Cohort A): A phase II, randomized, double-blind, placebo-controlled trial of lebrikizumab as monotherapy in patients with idiopathic pulmonary fibrosis. Am $J$ Respir Crit Care Med 2018; 197: A6167.

12 Maher TM, Kondoh Y, Corte TJ, et al. The RIFF Study (Cohort B): A phase II, randomized, double-blind, placebo-controlled trial of lebrikizumab in combination with pirfenidone in patients with idiopathic pulmonary fibrosis. Am J Respir Crit Care Med 2018; 197: A6168.

13 Rao E, Li D, Underwood S, et al. Generation of a tetravalent bispecific antibody against IL4 and IL13 for the treatment of idiopathic pulmonary fibrosis. MAbs 2012; 4: 15.

14 Soubrane C, Lin Y, Delfolie A, et al. Evaluation of the safety, tolerability, pharmacokinetics (PK) and pharmacodynamics (PD) of ascending repeated doses of SAR156597 in patients with idiopathic pulmonary fibrosis (IPF): a randomized, double-blind, placebo-controlled study (TDR11326). 18th International Colloquium on Lung and Airway Fibrosis (ICLAF), 2014. www.iclaf.com/Final_ICLAF_Program.pdf. Date last accessed: November 6, 2018. Date last updated: 2014.

15 Faffe DS, Whitehead T, Moore PE, et al. IL-13 and IL-4 promote TARC release in human airway smooth muscle cells: role of IL-4 receptor genotype. Am J Physiol 2003; 285: L907-L914.

16 Collard HR, Ryerson CJ, Corte TJ, et al. Acute exacerbation of idiopathic pulmonary fibrosis. An international working group report. Am J Respir Crit Care Med 2016; 194: 265-275.

17 Richeldi L, du Bois RM, Raghu G, et al. Efficacy and safety of nintedanib in idiopathic pulmonary fibrosis. $N$ Engl J Med 2014; 370: 2071-2082.

18 Noble PW, Albera C, Bradford WZ, et al. Pirfenidone in patients with idiopathic pulmonary fibrosis (CAPACITY): two randomised trials. Lancet 2011; 377: 1760-1769.

19 King TE Jr, Bradford WZ, Castro-Bernardini S, et al. A phase 3 trial of pirfenidone in patients with idiopathic pulmonary fibrosis. N Engl J Med 2014; 370: 2083-2092.

20 Idiopathic Pulmonary Fibrosis Clinical Research Network, Martinez FJ, de Andrade JA, et al. Randomized trial of acetylcysteine in idiopathic pulmonary fibrosis. N Engl J Med 2014; 370: 2093-2101.

21 Raghu G, Brown KK, Collard HR, et al. Efficacy of simtuzumab versus placebo in patients with idiopathic pulmonary fibrosis: a randomised, double-blind, controlled, phase 2 trial. Lancet Respir Med 2017; 5: 22-32.

22 Raghu G, Behr J, Brown KK, et al. Treatment of idiopathic pulmonary fibrosis with ambrisentan: a parallel, randomized trial. Ann Intern Med 2013; 158: 641-649. 